JOURNAL OF INTEGRAL EQUATIONS

AND APPLICATIONS

Volume 8, Number 4, Fall 1996

\title{
ON THE SOLUTION OF NONLINEAR VOLTERRA CONVOLUTION EQUATION WITH POWER NONLINEARITY
}

NIKOLAI K. KARAPETYANTS, ANATOLY A. KILBAS AND MEGUMI SAIGO

ABSTRACT. The Volterra nonlinear convolution integral equation

$$
\begin{gathered}
\varphi^{m}(x)=a(x) \int_{0}^{x} k(x-t) \varphi(t) d t+f(x) \\
0<x<d \leq \infty
\end{gathered}
$$

with $m>0, m \neq 1$, and real functions $a(x), k(u)$ and $f(x)$ is studied. Local estimates and asymptotic properties near zero for its solution $\varphi(x)$ are given, provided that $a(x), k(u)$ and $f(x)$ have power asymptotic behaviors near zero. The integral equation with a power kernel being solvable in closed form is considered.

1. Introduction. The Volterra nonlinear convolution integral equation

$$
\varphi^{m}(x)=a(x) \int_{0}^{x} k(x-t) \varphi(t) d t+f(x), \quad 0<x<d \leq \infty
$$

with $m \in \mathbf{R}$ arises in nonlinear theory of wave propagation [11] and water perlocation $[\mathbf{8}, \mathbf{1 8}]$. Such an equation with $m>0, m \neq 1$, was studied in $[\mathbf{1}, \mathbf{4}, \mathbf{6}, \mathbf{9}, \mathbf{1 6}, \mathbf{1 7}, \mathbf{1 8}]$ for $a(x)=1$ and in $[\mathbf{2}, \mathbf{3}, \mathbf{5}, \mathbf{7}]$ for the general case. It is proved that the solvability of equation (1) is different in the cases $m>1$ and $0<m<1$. In the former case the corresponding homogeneous equation, $f(x) \equiv 0$, can have a nontrivial solution, which was first proved in [21], and the papers above were devoted to investigate problems concerning the existence and uniqueness for the solution $\varphi(x)$ of the nonhomogeneous equation (1), the stability of such a solution and the method of successive approximation to construct this solution. Some results about the uniqueness of the solution of equation

Accepted for publication by the editors on June 1, 1996 .

Copyright @191996 Rocky Mountain Mathematics Consortium 
(1) with $a(x)=1$ and $m>1$ and continuous kernel $k(u)$ in the spaces of continuous and summable functions were given in $[\mathbf{1}, \mathbf{4}, \mathbf{9}]$. Such a problem for the homogeneous equation (1) with $a(x)=1, m<0$ and nonincreasing kernel $k(u)$ in the class of almost decreasing functions was studied in [10].

The existence of the solution for the equation

$$
\varphi^{m}(x)=a(x) \int_{0}^{x} \frac{\varphi(t)}{(x-t)^{1-\alpha}} d t+f(x), \quad 0<x<d \leq \infty,
$$

with $\alpha>0$ and the weak singular kernel $k(u)=u^{\alpha-1}$ for $0<\alpha<1$ was investigated in spaces of locally bounded and continuous functions in [12]. Asymptotic properties at zero of the solution $\varphi(x)$ of equation (2) with $m \in \mathbf{R}, m \neq 0,-1,-2, \ldots$, in the case when $a(x)$ and $f(x)$ have special asymptotics at zero were studied in $[13,14,15,19]$.

Our paper is devoted to the study of the local estimates and asymptotic properties for the solution $\varphi(x)$ of equation (1) with $m>0$, $\varphi^{m}(x)=e^{\pi m i} e^{m \log (|\varphi(x)|)}$ for $\varphi(x)<0$, and real functions $a(x), k(u)$ and $f(x)$. Section 2 deals with local behavior of the convolution

$$
(k * \varphi)(x)=\int_{0}^{x} k(x-t) \varphi(t) d t .
$$

In Section 3 we obtain the lower estimate for the solution $\varphi(x)$ of equation (1). Section 4 is devoted to a study of the asymptotic behavior at zero for the solution $\varphi(x)$ of equation (1), provided that the functions $a(x), k(u)$ and $f(x)$ have the asymptotic estimates

$$
\begin{aligned}
& a(x)=a x^{\mu}+o\left(x^{\mu}\right), \quad x \rightarrow+0, \\
& k(u)=k u^{\alpha-1}+o\left(u^{\alpha-1}\right), \quad u \rightarrow+0, \\
& f(x)=b x^{\nu}+o\left(x^{\nu}\right), \quad x \rightarrow+0
\end{aligned}
$$

for $a, k, b, \mu, \nu \in \mathbf{R}, a \neq 0, k \neq 0, b \neq 0$ and $\alpha>0$. An application to solve equation (1) in closed form is given in Sections 5 and 6.

2. Local behavior of convolution. Let $C(0, \infty)$ be the space of real valued continuous functions on $(0, \infty)$, and let $L_{1}^{\text {loc }}(0, \infty)$ be the space of all Lebesgue measurable functions belonging to $L_{1}(c, d)$ for all 
$c$ and $d$ such that $0 \leq c<d<\infty$. We denote by $C L_{\text {loc }}(0, \infty)$ the intersection of the spaces $C(0, \infty)$ and $L_{1}^{\text {loc }}(0, \infty)$ :

$$
C L_{\mathrm{loc}}(0, \infty) \equiv C(0, \infty) \bigcap L_{1}^{\mathrm{loc}}(0, \infty),
$$

and by $C L_{\text {loc }}^{+}(0, \infty)$ its subspace which consists of nonnegative functions in $C L_{\mathrm{loc}}(0, \infty)$. We note that a function in $C L_{\mathrm{loc}}(0, \infty)$ can have "singular" points only at zero and at infinity. The local behavior of the convolution (3) is given by the following statement.

Theorem 1. If $k(x), \varphi(x) \in C L_{\mathrm{loc}}(0, \infty)$, then $(k * \varphi)(x) \in$ $C L_{\mathrm{loc}}(0, \infty)$.

Proof. By $(7), k(x), \varphi(x) \in L_{1}(c, d)$ for any $c, d, 0<c<d<\infty$. Then, in accordance with Young's theorem (see, for example, [20, Theorem 1.4]), we find $(k * \varphi)(x) \in L_{1}(c, d)$ and

$$
\|(k * \varphi)(x)\|_{L_{1}(c, d)} \leq\|k\|_{L_{1}(0, d)}\|\varphi\|_{L_{1}(c, d)}
$$

and hence

$$
(k * \varphi)(x) \in L_{1}^{\text {loc }}(0, \infty)
$$

Now we prove that

$$
(k * \varphi)(x) \in C(0, \infty) .
$$

Let $x \in(0, \infty)$ and $\delta>0$. We have

$$
\begin{aligned}
(k * \varphi)(x+\delta)-(k * \varphi)(x)= & \int_{0}^{x+\delta} k(x+\delta-t) \varphi(t) d t \\
& -\int_{0}^{x} k(x-t) \varphi(t) d t \\
= & \int_{x}^{x+\delta} k(x+\delta-t) \varphi(t) d t \\
& +\int_{0}^{x / 2}[k(y+\delta)-k(y)] \varphi(x-y) d y \\
& +\int_{x / 2}^{x}[k(y+\delta)-k(y)] \varphi(x-y) d y \\
\equiv & I_{1}(\delta)+I_{2}(\delta)+I_{3}(\delta) .
\end{aligned}
$$


Since $k(x) \in L_{1}^{\text {loc }}(0, \infty)$ and $\varphi(x) \in C(0, \infty)$, for $I_{1}(\delta)$ and $I_{2}(\delta)$ we have the estimates:

$$
\left|I_{1}(\delta)\right| \leq \max _{x \leq t \leq x+\delta}|\varphi(t)| \int_{0}^{\delta}|k(y)| d y \longrightarrow 0, \quad \delta \longrightarrow+0
$$

and

$$
\left|I_{2}(\delta)\right| \leq \max _{x / 2 \leq t \leq x}|\varphi(t)| \int_{0}^{x / 2}|k(y+\delta)-k(y)| d y \longrightarrow 0, \quad \delta \longrightarrow+0 .
$$

Since $k(x) \in C(0, \infty)$, the function $k(y)$ is uniformly continuous on $[x / 2, x]$ for any $x \in(0, \infty)$, and hence:

$$
\max _{x / 2 \leq y \leq x}|k(y+\delta)-k(y)| \longrightarrow 0, \quad \delta \longrightarrow+0 .
$$

This implies from $\varphi(x) \in L_{1}^{\text {loc }}(0, \infty)$ that the estimate for $I_{3}(\delta)$ :

$$
\begin{gathered}
\left|I_{3}(\delta)\right| \leq\left(\max _{x / 2 \leq y \leq x}|k(y+\delta)-k(y)|\right) \int_{0}^{x / 2}|\varphi(y)| d y \longrightarrow 0 \\
\delta \longrightarrow+0 .
\end{gathered}
$$

It follows from (11)-(14) that, for all $x \in(0, \infty)$,

$$
\lim _{\delta \rightarrow+0}(k * \varphi)(x+\delta)=(k * \varphi)(x)
$$

and (10) is proved. Thus we obtain $(k * \varphi)(x) \in C L_{\mathrm{loc}}(0, \infty)$, and the theorem is proved.

3. Lower estimate for solution. Let us now investigate the lower estimate for the nonnegative solution $\varphi(x)$ of the nonlinear integral equation (1) with $m>1$.

Theorem 2. Let $m, \alpha, \mu \in \mathbf{R}$ such that

$$
m>1, \quad 0<\alpha \leq 1, \quad m+\alpha+\mu>1,
$$


and let the functions $a(x), k(x), f(x) \in C L_{\mathrm{loc}}^{+}(0, \infty)$ satisfy the conditions

$$
\begin{gathered}
a(x) \geq a x^{\mu}, \quad k(x) \geq k x^{\alpha-1}, \quad x \in(0, \infty), \\
f(x) \neq 0, \quad x \in(0, \varepsilon)
\end{gathered}
$$

with $a>0, k>0$ and $\varepsilon>0$. If equation (1) is solvable in the space $C L_{\mathrm{loc}}^{+}(0, \infty)$, then its solution $\varphi(x)$ satisfies the estimate

$$
\varphi(x) \geq\left(\frac{a k(m-1)}{\mu+\alpha+m-1}\right)^{1 /(m-1)} x^{(\mu+\alpha) /(m-1)} .
$$

Proof. In view of (16) and (1), we have

$$
\varphi^{m}(x) \geq a k x^{\mu} \int_{0}^{x} \frac{\varphi(t)}{(x-t)^{1-\alpha}} d t
$$

and, since $0<\alpha \leq 1$, we obtain

$$
\varphi^{m}(x) \geq a k x^{\mu+\alpha-1} \int_{0}^{x} \varphi(t) d t .
$$

We set

$$
y(x)=\int_{0}^{x} \varphi(t) d t
$$

Then $\varphi(x)=y^{\prime}(x)$, and we rewrite (18) in the form

$$
\left[y^{\prime}(x)\right]^{m} \geq a k x^{\mu+\alpha-1} y(x)
$$

or

$$
y^{\prime}(x) y^{-1 / m}(x) \geq(a k)^{1 / m} x^{(\mu+\alpha-1) / m} .
$$

Integrating the latter relation on $(0, x)$, and using the conditions $m+\alpha+\mu>1, m>1$ and (19), we arrive at the estimate

$$
y(x) \geq(a k)^{1 /(m-1)}\left(\frac{m-1}{\mu+\alpha+m-1}\right)^{m /(m-1)} x^{\{(\mu+\alpha) /(m-1)\}+1} .
$$


Substituting (21) into (18), we have

$$
\varphi^{m}(x) \geq\left(\frac{a k(m-1)}{\mu+\alpha+m-1}\right)^{m /(m-1)} x^{(\mu+\alpha) m /(m-1)} .
$$

From here we obtain relation (17). This completes the proof of the theorem.

Corollary 2.1. Let $m>1,0<\alpha \leq 1$ and $\mu \in \mathbf{R}$ such that $m+\alpha+\mu>1$, and let the functions $a(x), f(x) \in C L_{\mathrm{loc}}^{+}(0, \infty)$ satisfy the conditions

$$
a(x) \geq a x^{\mu}, \quad x \in(0, \infty), \quad f(x) \neq 0, \quad x \in(0, \varepsilon)
$$

with $a>0$ and $\varepsilon>0$. If equation (2) with $0<\alpha \leq 1$ is solvable in the space $C L_{\mathrm{loc}}^{+}(0, \infty)$, then its solution $\varphi(x)$ has the estimate $(17)$.

Corollary 2.2. Let $m>1$ and $\mu \in \mathbf{R}$ such that $m+\mu>0$, and let the functions $a(x), k(x), f(x) \in C L_{\mathrm{loc}}^{+}(0, \infty)$ satisfy the conditions

$$
\begin{gathered}
a(x) \geq a x^{\mu}>0, \quad k(x) \geq k>0, \quad x \in(0, \infty), \\
f(x) \neq 0, \quad x \in(0, \varepsilon)
\end{gathered}
$$

with $\varepsilon>0$. If equation (1) is solvable in the space $C L_{\mathrm{loc}}^{+}(0, \infty)$, then its solution $\varphi(x)$ satisfies the estimate

$$
\varphi(x) \geq\left(\frac{a k(m-1)}{\mu+m}\right)^{1 /(m-1)} x^{(\mu+1) /(m-1)} .
$$

Corollary 2.3. Let $m>1$, and let the functions $a(x), k(x)$, $f(x) \in C L_{\mathrm{loc}}^{+}(0, \infty)$ satisfy the conditions

$$
\begin{gathered}
a(x) \geq a>0, \quad k(x) \geq k>0, \quad x \in(0, \infty), \\
f(x) \neq 0, \quad x \in(0, \varepsilon)
\end{gathered}
$$

with $\varepsilon>0$. If equation (1) is solvable in the space $C L_{\mathrm{loc}}^{+}(0, \infty)$, then its solution $\varphi(x)$ satisfies the estimate

$$
\varphi(x) \geq\left(\frac{a k(m-1)}{m}\right)^{1 /(m-1)} x^{1 /(m-1)} .
$$


Remark 1. It follows from estimate (17) that if the conditions of Theorem 2 are satisfied and the solution of equation (1) has the asymptotic behavior, as $x \rightarrow 0$, of the form

$$
\varphi(x)=c x^{\gamma}+o\left(x^{\gamma}\right), \quad c \neq 0,
$$

then the upper estimate for $\gamma$,

$$
\gamma \leq \frac{\mu+\alpha}{m-1}
$$

necessarily must be valid. The exact value of $\gamma$ and a constant $c$ in (27) will be found in the next section.

4. Asymptotic behavior of solution at zero. Now we turn to treat the nonlinear convolution equation (1), provided that $m>0$, $m \neq 1$, and the functions $a(x), k(x), f(x) \in C L_{\mathrm{loc}}(0, \infty)$ satisfy the conditions (4)-(6). We suppose that equation (1) has a solution $\varphi(x) \in C L_{\mathrm{loc}}(0, \infty)$ and shall seek the asymptotic of this solution, as $x \rightarrow+0$, in the form (27) with unknown $c$ and $\gamma$. By using (4) and (5), it is directly verified that

$$
\begin{aligned}
a(x) \int_{0}^{x} k(x-t) \varphi(t) d t= & \frac{a k c \Gamma(\alpha) \Gamma(\gamma+1)}{\Gamma(\alpha+\gamma+1)} x^{\mu+\alpha+\gamma} \\
& +o\left(x^{\mu+\alpha+\gamma}\right), \quad x \rightarrow+0 .
\end{aligned}
$$

Therefore, on the basis of (6) and (27), we come from (1) to the asymptotic relation

$$
c^{m} x^{m \gamma} \sim \frac{a k c \Gamma(\alpha) \Gamma(\gamma+1)}{\Gamma(\alpha+\gamma+1)} x^{\mu+\alpha+\gamma}+b x^{\nu}, \quad x \rightarrow+0 .
$$

We consider three cases:

(i) $m \gamma<\mu+\alpha+\gamma$;

(ii) $m \gamma>\mu+\alpha+\gamma$;

(iii) $m \gamma=\mu+\alpha+\gamma=\nu$.

From (30) we obtain the following asymptotic relations, as $x \rightarrow+0$,

$$
c^{m} x^{m \gamma} \sim b x^{\nu} \quad \text { for (i), }
$$




$$
a k c \frac{\Gamma(\alpha) \Gamma(\gamma+1)}{\Gamma(\alpha+\gamma+1)} x^{\mu+\alpha+\gamma} \sim-b x^{\nu} \quad \text { for (ii) }
$$

and (30) for (iii). Then

$$
\begin{gathered}
\gamma=\nu / m, \quad c=b^{1 / m} \quad \text { for }(\mathrm{i}), \\
\gamma=\nu-\mu-\alpha, \quad c=-\frac{b \Gamma(\alpha+\gamma+1)}{a k \Gamma(\alpha) \Gamma(\gamma+1)} \quad \text { for (ii). }
\end{gathered}
$$

In case (iii),

$$
\gamma=\nu / m=\nu-\mu-\alpha
$$

and the number $c$ has to be a solution of the equation

$$
\xi^{m}-\frac{a k \Gamma(\alpha) \Gamma(\nu-\mu-\alpha+1)}{\Gamma(\nu-\mu+1)} \xi-b=0 .
$$

It follows from (33)-(35) that the three cases (i), (ii) and (iii) can be reclassified as

$$
\begin{gathered}
m(\nu-\mu-\alpha)<\nu \text { for (i); } m(\nu-\mu-\alpha)>\nu \text { for (ii); } \\
m(\nu-\mu-\alpha)=\nu \text { for (iii). }
\end{gathered}
$$

Then we obtain:

Theorem 3. Suppose $m>0, m \neq 1, \alpha>0$ and $\mu, \nu \in \mathbf{R}$ such that

$$
\max [\nu / m, \nu-\mu-\alpha]>-1
$$

Let the functions $a(x), k(x), f(x) \in C L_{\mathrm{loc}}(0, \infty)$ satisfy conditions (4)-(6), and let equation (1) have a solution $\varphi(x) \in C L_{\mathrm{loc}}(0, \infty)$.

If $m(\nu-\mu-\alpha) \neq \nu$, then the solution $\varphi(x)$ of equation (1) has the asymptotic behavior

$$
\varphi(x)=b^{1 / m} x^{\nu / m}+o\left(x^{\nu / m}\right), \quad x \rightarrow+0,
$$


when $m(\nu-\mu-\alpha)<\nu$, and

$$
\begin{aligned}
\varphi(x)= & -\frac{b \Gamma(\nu-\mu+1)}{a k \Gamma(\alpha) \Gamma(\nu-\mu-\alpha+1)} x^{\nu-\mu-\alpha} \\
& +o\left(x^{\nu-\mu-\alpha}\right), \quad x \rightarrow+0,
\end{aligned}
$$

when $m(\nu-\mu-\alpha)>\nu$.

If $m(\nu-\mu-\alpha)=\nu$, and if the algebraic equation (36) is solvable with $\xi=c$ being its solution, then the asymptotic of solution $\varphi(x)$ of the equation (1) is given by

$$
\begin{aligned}
\varphi(x) & =c x^{\nu / m}+o\left(x^{\nu / m}\right) \\
& =c x^{\nu-\mu-\alpha}+o\left(x^{\nu-\mu-\alpha}\right), \quad x \rightarrow+0 .
\end{aligned}
$$

Corollary 3.1. Suppose $m>0, m \neq 1, \alpha>0$ and $\mu, \nu \in \mathbf{R}$. Let the functions a $(x), k(x), f(x) \in C L_{\mathrm{loc}}(0, \infty)$ satisfy the conditions (4)-(6), and let equation (1) have a solution $\varphi(x) \in C L_{\mathrm{loc}}(0, \infty)$.

(i) If $\nu>\max [-m, m(\nu-\mu-\alpha)]$, then the asymptotic behavior of the solution $\varphi(x)$ of equation (1) is given by (39).

(ii) If $\mu+\alpha-1<\nu<m(\nu-\mu-\alpha)$, then the asymptotic behavior of the solution $\varphi(x)$ of equation (1) is given by (40).

(iii) If $m(\nu-\mu-\alpha)=\nu>-m$, then the asymptotic behavior of the solution $\varphi(x)$ of equation (1) is given by (41) with $c$ being given by a solution of (36).

Corollary 3.2. Suppose $m>0, m \neq 1, \alpha>0$ and $\mu, \nu \in \mathbf{R}$. Let the functions $a(x), k(x), f(x) \in C L_{\mathrm{loc}}(0, \infty)$ satisfy the conditions (4)-(6), and let equation (1) have a solution $\varphi(x) \in C L_{\mathrm{loc}}(0, \infty)$. If one of the following conditions holds:

$$
\begin{gathered}
m>1, \quad-m<\nu<\frac{(\mu+\alpha) m}{m-1}, \\
0<m<1, \quad \nu>\max \left[-m,-\frac{(\mu+\alpha) m}{1-m}\right],
\end{gathered}
$$


then the asymptotic behavior of the solution $\varphi(x)$, as $x \rightarrow+0$, of equation (1) is given by (39).

Corollary 3.3. Suppose $m>0, m \neq 1, \alpha>0$ and $\mu, \nu \in \mathbf{R}$. Let the functions $a(x), k(x), f(x) \in C L_{\mathrm{loc}}(0, \infty)$ satisfy the conditions (4)-(6), and let equation (1) have a solution $\varphi(x) \in C L_{\mathrm{loc}}(0, \infty)$. If one of the following conditions holds:

$$
\begin{aligned}
& 0<m<1, \quad \mu+\alpha-1<\nu<-\frac{(\mu+\alpha) m}{1-m}, \\
& m>1, \quad \nu>\max \left[\mu+\alpha-1, \frac{(\mu+\alpha) m}{m-1}\right],
\end{aligned}
$$

then the asymptotic behavior of the solution $\varphi(x)$, as $x \rightarrow+0$, of equation (1) is given by (40).

Remark 2. Let the conditions of Theorem 3 be valid, and equation (1) has the positive solution $\varphi(x) \in C L_{\text {loc }}^{+}(0, \infty)$. Then such a solution has the following asymptotics at zero: (i) (39) if $m(\nu-\mu-\alpha)<\nu$ and $b>0$, (ii) (40) if $m(\nu-\mu-\alpha)>\nu$ and $a k b<0$, (iii) (41) if $m(\nu-\mu-\alpha)=\nu$ and equation (36) has a positive solution $\xi=c>0$. In particular, if $a(x), k(x), b(x) \in C L_{\text {loc }}^{+}(0, \infty)$ with $a>0, k>0$, $b>0$ and $m>1$, the cases (i) and (iii) of Theorem 3 correspond to Remark 1.

5. Solution in closed form. The asymptotic (41) of the solution $\varphi(x)$ of equation (1) is led to the explicit solution of equation (2) with $\alpha>0$, when $a(x)=a x^{\mu}$ and $b(x)=b x^{\nu}$ with $m>0, m \neq 0,1$, and $a, b, \mu, \nu \in \mathbf{R}, a \neq 0, b \neq 0$ :

$$
\varphi^{m}(x)=\frac{a x^{\mu}}{\Gamma(\alpha)} \int_{0}^{x} \frac{\varphi(t)}{(x-t)^{1-\alpha}} d t+b x^{\nu}, \quad 0<x<\infty .
$$

It is directly verified that the function

$$
\varphi(x)=c x^{\nu / m}
$$


gives the solution $\varphi(x)$ of equation (46) provided that $m(\nu-\mu-\alpha)=\nu$, if equation (36) is solvable and $\xi=c$ is its solution. Thus we obtain the following result:

Theorem 4. Suppose $\alpha>0, m>0, m \neq 0,1$ and $\nu>-m$. Let the equation

$$
\xi^{m}-\frac{a \Gamma(\nu / m+1)}{\Gamma(\nu / m+\alpha+1)} \xi-b=0
$$

for $a, b \in \mathbf{R}, a \neq 0, b \neq 0$, be solvable and $\xi=c$ be its solution. Then the nonlinear integral equation

$$
\varphi^{m}(x)=\frac{a x^{-\alpha+\nu-\nu / m}}{\Gamma(\alpha)} \int_{0}^{x} \frac{\varphi(t)}{(x-t)^{1-\alpha}} d t+b x^{\nu}, \quad 0<x<\infty
$$

is solvable and its solution $\varphi(x)$ has the form (47).

Corollary 4.1. Suppose $\alpha>0$ and $m>0, m \neq 0,1$. Let the equation

$$
\xi^{m}-\frac{a}{\Gamma(\alpha+1)} \xi-b=0
$$

for $a, b \in \mathbf{R}, a \neq 0, b \neq 0$, be solvable and $\xi=c$ be its solution. Then the nonlinear integral equation

$$
\varphi^{m}(x)=\frac{a x^{-\alpha}}{\Gamma(\alpha)} \int_{0}^{x} \frac{\varphi(t)}{(x-t)^{1-\alpha}} d t+b, \quad 0<x<\infty
$$

is solvable, and its solution $\varphi(x)$ is the constant

$$
\varphi(x)=c .
$$

In conclusion, we discuss the uniqueness of solution (47) of equation (49). If $0<m<1$ and the algebraic equation (48) has a unique solution $\xi=c$, then it follows from the results in $[\mathbf{1 2}]$ that the solution (47) of equation (49) is unique in the space $C(0, \infty)$. Since 
$\varphi(x)=c x^{\nu / m} \in L_{\text {loc }}(0, \infty)$ for $\nu>-m, \varphi(x)$ belongs to the space $C L_{\text {loc }}(0, \infty)$. If, additionally, $c>0$, then this solution is unique in $C^{+}(0, \infty)$ and belongs to $C L_{\mathrm{loc}}^{+}(0, \infty)$.

In the case $m>1$ the uniqueness of solution (47) of equation (49) is not clear. For example, we consider equation (49) with $m=2$ :

$$
\varphi^{2}(x)=\frac{a x^{-\alpha+\nu / 2}}{\Gamma(\alpha)} \int_{0}^{x} \frac{\varphi(t)}{(x-t)^{1-\alpha}} d t+b x^{\nu}, \quad 0<x<\infty
$$

for $\alpha>0, \nu>-2$ and $a, b \in \mathbf{R}, a \neq 0, b \neq 0$. Equation (48) takes the form

$$
\Gamma(\nu / 2+\alpha+1) \xi^{2}-a \Gamma(\nu / 2+1) \xi-b \Gamma(\nu / 2+\alpha+1)=0 .
$$

If the discriminant

$$
D \equiv a^{2} \Gamma^{2}(\nu / 2+1)+4 b \Gamma^{2}(\nu / 2+\alpha+1)>0,
$$

then equation (54) has two solutions

$$
\xi=c_{1}=\frac{a \Gamma(\nu / 2+1)+\sqrt{D}}{2 \Gamma(\nu / 2+\alpha+1)}, \quad \xi=c_{2}=\frac{a \Gamma(\nu / 2+1)-\sqrt{D}}{2 \Gamma(\nu / 2+\alpha+1)}
$$

and the one for $D=0$

$$
\xi=c_{3}=\frac{a \Gamma(\nu / 2+1)}{2 \Gamma(\nu / 2+\alpha+1)}
$$

It follows from Theorem 4 that equation (54) has two solutions in the space $C L_{\text {loc }}(0, \infty)$

$$
\varphi(x)=c_{1} x^{\nu / 2}, \quad \varphi(x)=c_{2} x^{\nu / 2}
$$

when $D>0$ and the one

$$
\varphi(x)=c_{3} x^{\nu / 2}
$$

when $D=0$.

If $a>0$ and $b>0$, then $\sqrt{D}>a \Gamma(\nu / 2+1)$, and hence $c_{1}>0, c_{2}<0$. Therefore, equation (53) has a positive solution

$$
\varphi(x)=c_{1} x^{\nu / 2}
$$


which belongs to the space $C L_{\text {loc }}^{+}(0, \infty)$. If $a>0$ and $D=0$, equation (53) has the positive solution (59). In the case $a>0, b<0$ and $D>0$ equation (53) has two positive solutions given in (58).

So we obtain the result.

Theorem 5. Let $\nu>-2$, and let $D$ be given by (55). If $D \geq 0$, then the nonlinear integral equation (53) is solvable in the space $C L_{\mathrm{loc}}(0, \infty)$ and has two solutions (58) when $D>0$ and the one (59) when $D=0$. If $D \geq 0$ and $a>0$, then equation (53) is solvable in the space $C L_{\mathrm{loc}}^{+}(0, \infty)$ and has two positive solutions $(58)$ when $D>0$ and $b<0$, and the one (59) or (60) when $D=0$ or $D>0$ and $b>0$, respectively.

Remark 3. By Theorem 4, the nonlinear integral equation (49) is solvable together with the algebraic equation (48). In applications it is important to know positive solutions of equation (49). According to Theorem 5, equation (53), being equation (49) with $m=2$, can have one or two positive solutions together with the corresponding square equation (54). In the next section we shall prove that such a result is also valid for the integral and algebraic equations (49) and (48).

6. Positive solutions of algebraic and integral equations. We first consider the algebraic equation of the form (48):

$$
\xi^{m}-d \xi-b=0
$$

with $m>0, m \neq 1$ and $d, b \in \mathbf{R}, d \neq 0, b \neq 0$ and investigate positive solvability of such an equation by using the properties of the function

$$
f(\xi)=\xi^{m}-d \xi-b .
$$

If $d<0$ and $b<0$, then $f(\xi)>0$ for $\xi \geq 0$, and the equation (61) does not have positive solutions.

If $d<0$ and $b>0$, then $f(0)=-b<0, f^{\prime}(\xi)=m \xi^{m-1}-d>0$ for $\xi>0$, and hence equation (61) has a unique positive solution $\xi=c_{1}>0$.

When $d>0$, the circumstances become complex. In fact, $f(\xi)$ has the positive extremal point

$$
\xi=c_{0}=\left(\frac{d}{m}\right)^{1 /(m-1)},
$$


for which

$$
\begin{gathered}
f^{\prime}\left(c_{0}\right)=0, \quad E \equiv f\left(c_{0}\right)=(1-m)\left(\frac{d}{m}\right)^{m /(m-1)}-b, \\
f^{\prime \prime}\left(c_{0}\right)=m(m-1) c_{0}^{m-2}
\end{gathered}
$$

and

$$
f^{\prime \prime}\left(c_{0}\right)>0, \quad m>1, \quad f^{\prime \prime}\left(c_{0}\right)<0, \quad 0<m<1 .
$$

When $d>0$ and $b<0$, then $f(0)=-b>0$. When $0<m<1$, then $E>0$, and in accordance with $(65), f^{\prime}(\xi)>0$ for $0<\xi<c_{0}$ and $f^{\prime}(\xi)<0$ for $\xi>c_{0}$. Hence, equation (61) has a unique positive solution $\xi=c_{1}>c_{0}$. When $m>1, f^{\prime}(\xi)<0$ for $0<\xi<c_{0}$ and $f^{\prime}(\xi)>0$ for $\xi>c_{0}$, and therefore equation (61) does not have positive solutions if $E>0$, has a unique positive solution $\xi=c_{0}$ if $E=0$, and two positive solutions $\xi=c_{2}$ and $\xi=c_{3}, 0<c_{2}<c_{0}<c_{3}$, if $E<0$.

When $d>0$ and $b>0$, then $f(0)=-b<0$, and, similarly to the above, we prove that equation (61) has a unique positive solution $\xi=c_{1}>c_{0}$ if $m>1$. When $0<m<1$, equation (61) does not have positive solutions if $E<0$, has a unique positive solution $\xi=c_{0}$ if $E=0$ and two positive solutions $\xi=c_{2}$ and $\xi=c_{3}, 0<c_{2}<c_{0}<c_{3}$, if $E>0$.

Thus we obtain the result:

Theorem 6. Let $m>0, m \neq 1, d, b \in \mathbf{R}, d \neq 0, b \neq 0$, and let $E$ and $c_{0}$ be given by (64) and (63). Equation (61)

(i) does not have positive solutions if either (a) $d<0, b<0$ or (b) $d>0, b<0, m>1, E>0$ or (c) $d>0, b>0,0<m<1, E<0$;

(ii) has a unique positive solution $\xi=c_{1}>0$ if (d) $d<0, b>0$; $\xi=c_{1}>c_{0}>0$ if either (e) $d>0, b<0,0<m<1$ or (f) $d>0$, $b>0, m>1 ; \xi=c_{0}>0$ if $E=0$ and either $(\mathrm{g}) d>0, b<0, m>1$ or (h) $d>0, b>0,0<m<1$;

(iii) has two positive solutions $\xi=c_{2}$ and $\xi=c_{3}, 0<c_{2}<c_{0}<c_{3}$, if either (i) $d>0, b<0, m>1, E<0$ or (j) $d>0, b>0,0<m<1$, $E>0$. 
Let us consider the function of $\xi: g(\xi)=\xi^{m}-d \xi^{q}-b$ for $q>0$ and $m>0, m \neq q$. Let

(66) $\bar{c}_{0}=\left(\frac{q d}{m}\right)^{1 /(m-q)}$ and $F \equiv g\left(\bar{c}_{0}\right)=\frac{q-m}{q}\left(\frac{q d}{m}\right)^{m /(m-q)}-b$.

Corollary 6.1. Let $q>0, m>0, m \neq q, d, b \in \mathbf{R}, d \neq 0, b \neq 0$, and let $F$ and $\bar{c}_{0}$ be given by (66). Then the equation $g(\xi)=0$

(i) does not have positive solutions if either (a) $d<0, b<0$ or (b) $d>0, b<0, m>q, F>0$ or (c) $d>0, b>0,0<m<q, F<0$;

(ii) has a unique positive solution $\xi=\bar{c}_{1}>0$ if (d) $d<0, b>0$; $\xi=\bar{c}_{1}>\bar{c}_{0}>0$ if either (e) $d>0, b<0,0<m<q$ or (f) $d>0$, $b>0, m>q ; \xi=\bar{c}_{0}>0$ if $F=0$ and either $(\mathrm{g}) d>0, b<0, m>q$ or (h) $d>0, b>0,0<m<q$;

(iii) has two positive solutions $\xi=\bar{c}_{2}$ and $\xi=\bar{c}_{3}, 0<\bar{c}_{2}<\bar{c}_{0}<\bar{c}_{3}$, if either (i) $d>0, b<0, m>q, F<0$ or (j) $d>0, b>0,0<m<q$, $F>0$.

Corollary 6.1 follows from Theorem 6 since after replacing $\xi$ by $\xi^{1 / q}$ the equation $g(\xi)=0$ is reduced to equation (61) with $m$ being replaced by $m / q$.

From Theorems 4 and 6 we obtain the statement similar to Theorem 5 .

Theorem 7. Let $\alpha>0, m>0, m \neq 1, \nu>-m, a, b \in \mathbf{R}, a \neq 0$, $b \neq 0$, and let

$$
c_{0}=\left(\frac{a \Gamma(\nu / m+1)}{m \Gamma(\nu / m+\alpha+1)}\right)^{1 /(m-1)}, \quad E=(1-m) c_{0}^{m}-b .
$$

If either of the conditions (a) $a<0, b<0$ or (b) $a>0, b<0$, $m>1, E>0$ or (c) $a>0, b>0,0<m<1, E<0$ is valid, then the nonlinear integral equation (49) is not solvable in the space $C L_{\text {loc }}^{+}(0, \infty)$.

(i) If either (d) $a<0, b>0$ or (e) $a>0, b<0,0<m<1$ or (f) $a>0, b>0, m>1$, then equation (49) has the positive solution

$$
\varphi(x)=c_{1} x^{\nu / m}
$$


where $\xi=c_{1}$ is a unique positive root of the algebraic equation (48): $c_{1}>0$ for the case (d) and $c_{1}>c_{0}>0$ for the case (e) or (f).

(ii) If $E=0$ and either (g) $a>0, b<0, m>1$ or (h) $a>0, b>0$, $0<m<1$, then the equation (49) has the positive solution

$$
\varphi(x)=c_{0} x^{\nu / m}
$$

where $\xi=c_{0}$ is a unique positive root of equation (48).

(iii) If either (i) $a>0, b<0, m>1, E<0$ or (j) $a>0, b>0$, $0<m<1, E>0$, then equation (49) has two positive solutions

$$
\varphi(x)=c_{2} x^{\nu / m}, \quad \varphi(x)=c_{3} x^{\nu / m},
$$

where $\xi=c_{2}$ and $\xi=c_{3}, 0<c_{2}<c_{0}<c_{3}$ are two positive roots of equation (48).

Acknowledgments. This work was initiated during the second author's visit to Fukoka University as Research Fellow in 1995. The work is supported partly by a Science Research Promotion Fund from the Japan Private School Promotion Foundation.

\section{REFERENCES}

1. S.N. Askhabov, Integral equations of convolution type with power nonlinearity, Colloq. Math. 62 (1991), 49-65.

2. S.N. Askhabov and M.A. Betilgiriev, Nonlinear convolution type equations, Seminar Analysis, Karl Weierstrass Inst. Math., Berlin, 1990.

3. - Nonlinear convolution integral equations with almost increasing kernels in cones, Differential Equations 27 (1991), 234-242.

4. S.N. Askhabov, N.K. Karapetyants and A.Ya. Yakubov, A nonlinear equation of convolution type, Differentsial'nye Uravneniya 22 (1986), 1606-1609 (Russian).

5. - Integral equations of convolution type with a power nonlinearity and their systems, Soviet Math. Dokl. 41 (1990), 323-327.

6. P.J. Bushell, On a class of Volterra and Fredholm non-linear integral equations, Math. Proc. Camb. Phil. Soc. 79 (1976), 329-335.

7. P.J. Bushell and W. Okrasinski, Nonlinear Volterra integral equations and the Apery identities, Bull. London Math. Soc. 24 (1992), 478-484.

8. J. Gonserzewicz, H. Marcinkowska, W. Okrasinski and K. Tabitz, On the perlocation of water from a cylindrical reservoir into the surrounding soil, Zastosow Math. 16 (1978), 249-261. 
9. N.K. Karapetyants, On a class of nonlinear equations of convolution type, Sci. Proc. Jubilee Sem. Bound. Value Problems, Minsk, 1985, 158-161 (Russian).

10. N.K. Karapetyants, A.Ya. Yakubov, A convolution equation with power nonlinearity of negative order, Soviet Math. Dokl. 44 (1992), 517-520.

11. J.J. Keller, Propagation of simple nonlinear waves in gas field tubes with friction, Z. Angew. Math. Phys. 32 (1981), 170-181.

12. A.A. Kilbas, A nonlinear Volterra integral equation with a weak singularity, Notes of Russian People's Friendship University 2 (1994), 210-224.

13. A.A. Kilbas and M. Saigo, On asymptotic solutions of nonlinear and linear Abel-Volterra integral equations, in Investigations in Jack's lemma and related topics, Sūrikaisekikenkyūsho Kōkyūroku 821 (1994), 91-111.

14. - On solution of integral equation of Abel-Volterra type, Differential Integral Equations 8 (1995), 993-1011.

15. A.A. Kilbas, M. Saigo and R. Gorenflo, On asymptotic solution of nonlinear Abel-Volterra integral equations with quasipolynomial free term, J. Fractional Calculus 8 (1995), 75-93.

16. W. Okrasinski, On the existence and uniqueness of nonnegative solution of a certain nonlinear convolution equation, Ann. Polon. Math. 36 (1979), 61-72.

17. - On a nonlinear convolution equation occurring in the theory of water perlocation, Ann. Polon. Math. 37 (1980), 223-229.

18. - Nonlinear Volterra equations and physical applications, Extracta Math. 4 (1989), 51-80.

19. M. Saigo and A.A. Kilbas, On asymptotic solutions of nonlinear and linear Abel-Volterra integral equations II, in Investigations in Jack's lemma and related topics, Sūrikaisekikenkyūsho Kōkyūroku 821 (1994), 112-129.

20. S.G. Samko, A.A. Kilbas and O.I. Marichev, Fractional integrals and derivatives. Theory and applications, Gordon and Breach, Yverdon et alibi, 1993.

21. W.R. Schneider, The general solution of a non-linear integral equation of convolution type, Z. Angew. Math. Phys. 33 (1982), 140-142.

Department of Mathematics, Rostov State University, Rostov-on-Don, Russia

Department of Mathematics and Mechanics, Belarusian State UniverSity, Minsk 220050, Belarus

Department of Applied Mathematics, Fukuoka University, Fukuoka 81480, JAPAN 